\title{
Comparison of T-SPOT TB and Tuberculin Skin Test in Children with Tuberculosis of Intrathoracic Lymph Nodes
}

\author{
Svetlana Atanasova Velizarova \\ Department of Pulmonary Diseases, Medical University, Sofia 1432, Bulgaria
}

\begin{abstract}
TB (Tuberculosis) of intrathoracic lymph nodes is the most common form of tuberculosis disease in childhood. In Bulgaria, about $67 \%-70 \%$ of all newly diagnosed children patients are with this diagnosis. The purpose of this study was to determine the diagnostic capabilities of the T-SPOT TB in this form of TB disease. In a period of one year, 50 children aged 0-12 years treated in our hospital with tuberculosis of lung lymph nodes were examined. The diagnosis was set based on history, clinical, microbiological and radiological check. T-SPOT TB (ELISPOT TB test) and the TST (tuberculin skin test) were performed simultaneously on the studied children. The relationship between immunological samples TST and T-SPOT TB (presence of BCG vaccination and contact with adult TB patient). One child had TST negative samples, $32 \%$ had normergic reactions to $15 \mathrm{~mm} .66 \%$ had hyperergic above $15 \mathrm{~mm}$. In a study with T-SPOT TB, $28 \%$ showed negative results and $72 \%$ showed positive.
\end{abstract}

Key words: IGRA tests, TST, T-SPOT TB, childhood TB

\section{Introduction}

The BCG (Bacille Calmette-Guerin) vaccine, discovered by Calmete and Guerin in 1921, was a colossal step forward for the immune prophylaxis of tuberculosis disease. Its mass implementation after 1948 lead to decreased incidence in Europe. Things changed in 1980-1990, when the HIV (human immunodeficiency virus) was discovered. It turned out that tuberculosis is an opportunistic disease in patients with AIDS (acquired immunodeficiency syndrome). Due to globalization and uncontrolled migration, the tuberculosis disease control is already very difficult.

It was necessary to find antigens that are strictly specific only to tuberculosis bacteria and are absent in BCG vaccine or atypical mycobacteria. This became possible after deciphering the tuberculosis bacterium genome. These specific antigens are ESAT-6 (early

Corresponding author: Svetlana Atanasova Velizarova, M.D., research fields: immunology, tuberculosis, BCG vaccination and pulmonary diseases in childhood. E-mail: velizarovasv@gmail.com; sv_velizarova@abv.bg. secreted antigen target 6) and CFP-10 (culture filtrate protein 10). These antigens are localized in the gene differentiated as RD 1 (region of deletion 1) segment of Mycobacterium tuberculosis. These two proteins are related to tuberculosis bacterium virulence and its cytotoxicity. They are directly responsible for the specific T-cell immune response [1-3].

In countries with mandatory BCG vaccination such as Bulgaria, the most common form of tuberculosis disease is tuberculosis of intrathoracic lymph nodes. It was found that $67 \%-70 \%$ of the children aged below 14 years had tuberculosis disease. The BCG vaccine manufacturers think that even after massive household contact, when the BCG vaccine is appropriately done, severe hematogenic forms of tuberculosis don't develop [4, 5].

The purpose of the present study was to compare the diagnostic capabilities of TST and T-SPOT TB in children with tuberculosis of intrathoracic lymph nodes. 


\section{Materials and Methods}

\subsection{Participants in the Study}

Children admitted for examination at "University Clinic of Pulmonary Diseases in Children” during 2009/2010 period were included in the study. All children have been referred to the clinic by pulmonary diseases dispensaries or by GP (general practitioner) with suspicion for tuberculosis disease or LTBI (latent tuberculosis infection). After conducted examinations, all children were diagnosed with tuberculosis of tracheobronchial lymph nodes prior to their inclusion in the study. The diagnosis was made according to relevant diagnostic algorithm described below.

\subsection{Inclusion Criteria}

(1) Case history;

(2) Epidemiological inquiry-data for cases of tuberculosis patients in the family or in the close circle of the child;

(3) Signs and symptoms of active tuberculosis disease;

(4) X-ray examination of lungs - data for enlarged intrathoracic lymph nodes.

\subsection{Investigations for Tuberculosis of Intrathoracic Lymph Nodes}

(1) Gastric lavage water was taken in all children and it was examined for tuberculosis bacteria directly and on culture;

(2) Mantoux tuberculin test was performed on left forearm, strictly intradermally with 5 IU Bulgarian PPD (purified protein derivative) tuberculin. The results were accounted after 72 hours. They were divided in three groups: infiltrate size $0-5 \mathrm{~mm}$-negative, infiltrate size 6-14 mm-normergy and above 15 mm-hyperergy;

(3) The presence or absence of BCG scar on the left shoulder was accounted in all children (mandatory BCG vaccination has been performed in Bulgaria since 1960);
(4) The presence of granulomatous enlarged lymph nodes was accounted on the performed X-ray examination and median tomography.

\subsection{Laboratory Analysis}

T-SPOT TB was examined in central immunological laboratory at "Alexandrovska" hospital according to recommendations of Oxford Immunotec, Abingdon, UK. All procedures, including also results interpretation, were performed according to the recommendations of the manufacturer. The spot-forming cells' values were counted with a microscope. Results were accounted according to Oxford Immunotec standards [6].

\subsection{Statistical Analysis}

Statistical analysis was made with SPSS 17 . The percentage ratio was examined in both tests at different diagnostic criteria. Concordance percentage and likelihood ratio were also examined.

\section{Results}

Fifty children diagnosed with tuberculosis of tracheobronchial lymph nodes treated at the clinic during the 2009/2010 period were examined. The children were aged from 4 months to 12 years. All were Bulgarian citizens.

Twenty seven children were girls and twenty three were boys. The children were divided into three age groups. Half of the children were aged 0 to 3 years, 17 children were aged 4 to 7 years and 8 children were elder than 8 years. All children were checked for presence of BCG scar on the left shoulder that is the only sign for exactly performed BCG vaccination. In 31 children (62\%), BCG vaccination scar was not found and 19 (38\%) had BCG scar. These results give us reason to think that there are lapses in our vaccination program.

Large part of the children, 37 (74\%) have had contacts with adults with tuberculosis disease with established excretion of Mycobacterium tuberculosis, 
which proves the direct link between development of tuberculosis in children and contact with adult with tuberculosis disease in the close circle of the child.

In 10 children (20\%), we had tuberculosis bacteria excretion directly or in culture, which is higher than the average for the country $14 \%-16 \%$.

Only one of the examined children was negative for TST, 16 children (32\%) had infiltrate size 6-14 mm. And the largest number of children, 33 (66\%) had infiltrate size above $15 \mathrm{~mm}$, which is accepted for hyperergy according to criteria for the Bulgarian population.

With T-SPOT TB, 14 children (28\%) had negative reactions, total positive were $36(72 \%)$ as 4 (8\%) were positive to ESAT-6, 10 (20\%) were positive to CFP-10 and 22 (44\%) reacted positive to both antigens, which shows that significant exposition to the infection is needed before developing the disease.

The mean age of the examined children was $3.8 \pm$ 2.9. The mean TST size was $15.1 \pm 4.6 \mathrm{~mm}$. With T-SPOT TB, the mean spot count for ESAT- 6 was 14.6 $\pm 14.5 \mathrm{~mm}$ and for CFP-10 was $18.6 \pm 15.9 \mathrm{~mm}$.

In the youngest age group, 11 (44\%) reacted to TST with infiltrate size 6-14 $\mathrm{mm}$ and 14 (56\%) with infiltrate size above $15 \mathrm{~mm}$, as the age increases the relative share of children who reacted with infiltrate size above $15 \mathrm{~mm}$ increases. With T-SPOT TB the relative share of negative reactions decreases with age. In most negative reactions, nine (36\%) are in the group aged 0-3 years. Valid correlation between age and reaction to T-SPOT TB is statistically found, likelihood ratio-21,089 df6—P-0.002 [7, 8]( Fig. 1).

There was no large difference in the reactions to TST and T-SPOT TB regarding the presence or absence of BCG scar. The relative share of children with infiltrate size above $15 \mathrm{~mm}$ without BCG scar was $64.5 \%$ and with BCG scar was $68.4 \%$. With $\mathrm{T}$ SPOT TB in children without BCG scar, $71 \%$ had positive reaction and in children with BCG scar $73.7 \%$ were positive, which shows that BCG vaccination doesn't influence T-SPOT TB, which is understandable since allergens are absent in BCG vaccine [9].

Fourteen children (37.8\%) who had contacts with adults with tuberculosis disease reacted to TST with infiltrate size 6-14 mm and twenty two children (59.5\%) had infiltrate size above $15 \mathrm{~mm}$. Totally, the children with such contacts were $74 \%$ of the examined children, which shows the direct adult-child link of the infection.

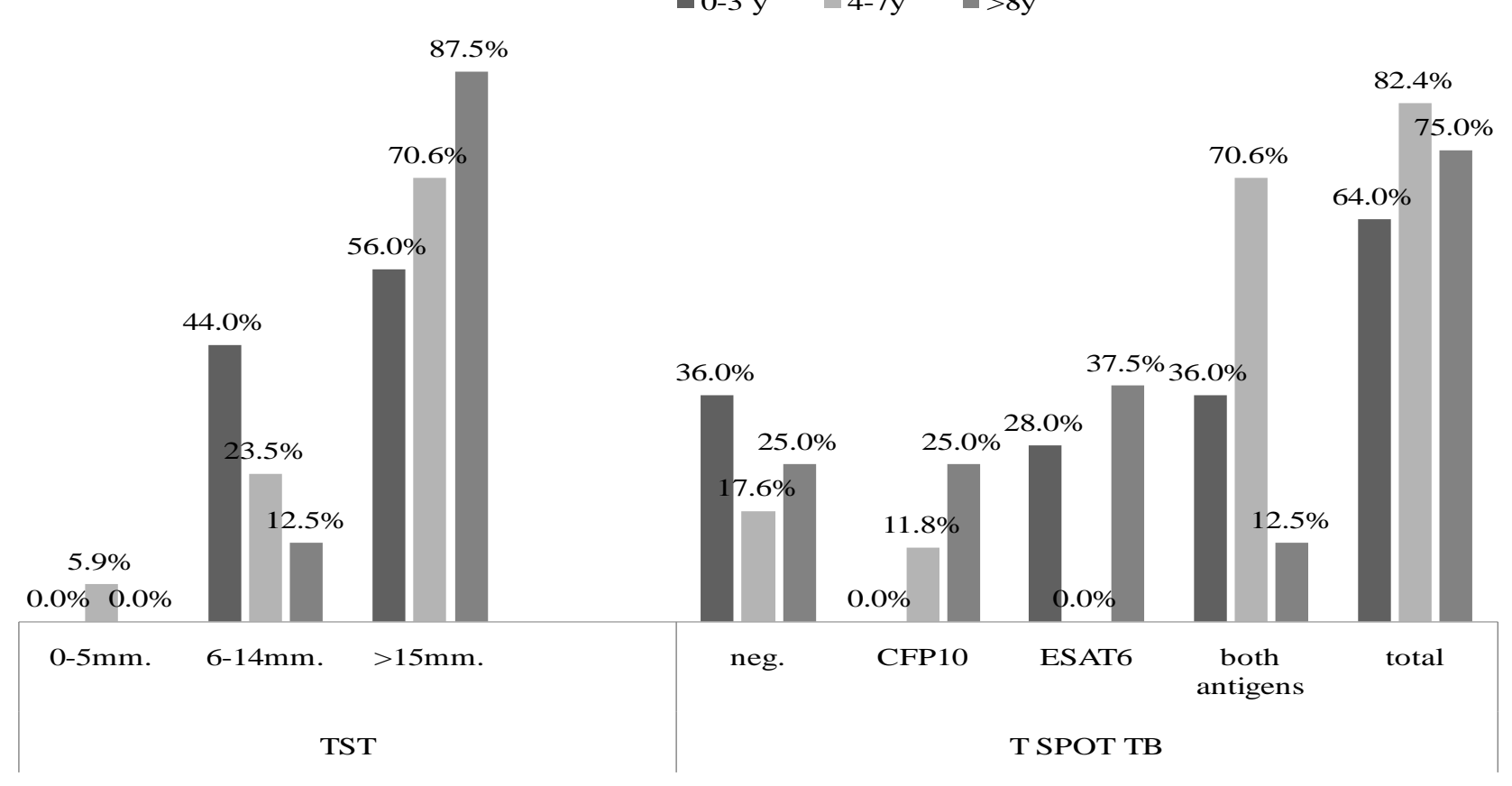

Fig. 1 Relative share of children who reacted to both tests by ages. 
With T-SPOT TB, 12 (32.4\%) children with contacts reacted negative [10].

In children with positive bacterial culture samples, $70 \%$ were with hyperergic tuberculin test, equal number reacted positive also with T-SPOT TB (Fig. 2).
The most interesting thing for us was the relationship between the two immunologic tests. When using only TST, 33 (66\%) are with infiltrate size above $15 \mathrm{~mm}$, when using only T-SPOT TB, 36 (72\%) give positive results. Of all examined, only one child was negative to both TST and T-SPOT TB (Fig. 3).

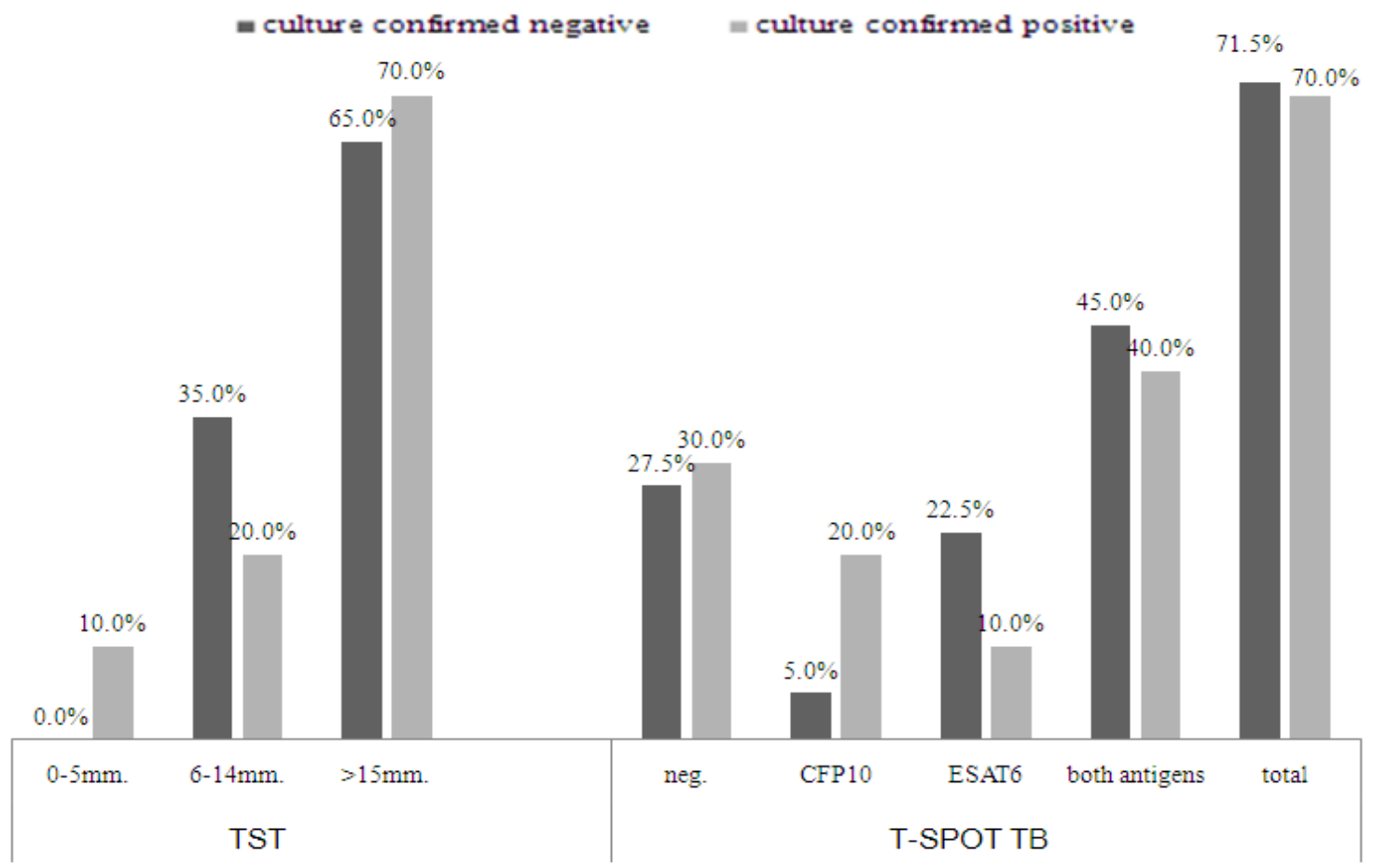

Fig. 2 Reactions to both tests by culture confirmed Mycobacterium tuberculosis.

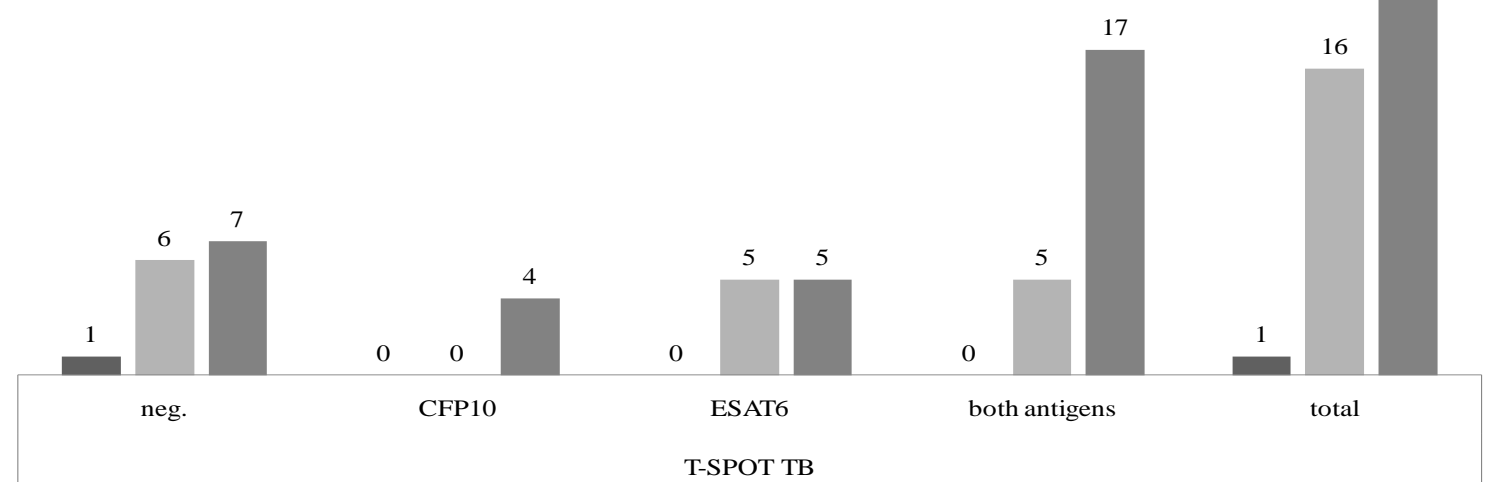

Fig. 3 Relationship between both examined tests. 
When using both tests, we have 98\% diagnostic success, which is confirmed by other authors. The concordance percentage is $80 \%$, which suggests good correlational relationship between the two tests [11-13].

\section{Discussion and Conclusions}

From the obtained results, it can be assumed that in children with diagnostically proven active tuberculosis (the most common form in childhood-tuberculosis of intrathoracic lymph nodes), TST has greater diagnostic value, since we had negative tuberculin test only in one child, while with T-SPOT TB, 14 children had negative results. It is noteworthy that the largest relative share of negative results was present in the youngest age group below 3 years, which is supported by many authors with the hypothesis that in this age group, the effector T-lymphocytes level is quantitatively low and this reflects to T-SPOT TB reactions. Many authors think that IGRA (interferon- $\gamma$ release assays) tests correlate to the degree of exposition, which is lowest in early childhood age [14-16]. The use of T-SPOT TB is recommended in cases of TST with size 6-14 $\mathrm{mm}$, that is considered normal in countries with mandatory BCG vaccination. In 16 children with normergic tuberculin test, 10 had positive results with T-SPOT TB. This once again proves that the use of both tests gives us very high diagnostic success result. In the conducted study, at which only $20 \%$ of the children had tuberculosis bacteria in gastric lavage, we proved that the use of both immunologic tests, together with the established diagnostic algorithm for tuberculosis is sufficient to make the right diagnosis $[17,18]$.

Based on the presented results, we can make the following conclusions:

(1) The diagnostic capabilities of T-SPOT TB and TST in tuberculosis of tracheobronchial lymph nodes are equal;

(2) T-SPOT TB test can be successfully used in a complex for diagnosing MTB infection in BCG-vaccinated children;
(3) When using both tests, we have 98\% diagnostic success.

\section{References}

[1] Lalvani, A. 2007. "Diagnosing Tuberculosis Infection in the 21st Century: New Tools to Tackle an Old Enemy.” Chest. 131 (6): 1898-906.

[2] Jones, C., Whittaker, E., Bamford, A., and Kampmann, B. 2011. "Immunology and Pathogenesis of Childhood TB." Paediatr. Respir. Rev. 12 (1): 3-8.

[3] Ling, D., Zwerling, A. A., Steingart, K., and Pai, M. 2011. "Immune-Based Diagnostics for TB in Children: What is the Evidence?” Paediatr. Respir. Rev. 12 (1): 9-15.

[4] Mahan, C. S., Johnson, D. F., Curley, C., and van der Kuyp, F. 2011. "Concordance of a Positive Tuberculin Skin Test and an Interferon $\gamma$ Release Assay in Bacille Calmette-Guérin Vaccinated Persons." Int. J. Tuberc. Lung Dis. 15 (2): 174-8.

[5] Kimura, M., Comstock, G. W., and Mori, T. 2005. "Comparison of Erythema and Induration as Results of Tuberculin Tests.” Int. J. Tuberc. Lung Dis. 9 (8): 853-7.

[6] Oxford Immunotec. "T-SPOT.TB: An Aid in the Diagnosis of Tuberculosis Infection.” Accessed March 20, 2013. http://www.oxfordimmunotec.com/8-uk.

[7] Pavic, I., Topic, R. Z., Raos, M., Aberle, N., and Dodig, S. 2011. "Interferon- $\gamma$ Release Assay for the Diagnosis of Latent Tuberculosis in Children Younger than 5 Years of Age.” Pediatr. Infect. Dis. J. 30 (10): 866-70.

[8] Debord, C., De Lauzanne, A., Gourgouillon, N., Guerin-El Khourouj, V., Pedron, B., Gaudelus, J., Faye, A., and Sterkers, G. 2011. "Interferon- $\gamma$ Release Assay Performance for Diagnosing Tuberculosis Disease in 0-to 5-Year-Old Children” Pediatr. Infect. Dis. J. 30 (11): 995-7.

[9] van Zyl-Smit, R. N., Zwerling, A., Dheda, K., and Pai, M. 2009. "Within-Subject Variability of Interferon- $\gamma$ Assay Results for Tuberculosis and Boosting Effect of Tuberculin Skin Testing: A Systematic Review." PLoS One 4 (12): e8517.

[10] Arend, S. M., Thijsen, S. F., Leyten, E. M., Bouwman, J. J., Franken, W. P., Koster, B. F., Cobelens, F. G., van Houte, A. J., and Bossink, A. W. 2007. "Comparison of Two Interferon-Gamma Assays and Tuberculin Skin Test for Tracing Tuberculosis Contacts.” Am. J. Respir. Crit. Care. Med. 175 (6): 618-27.

[11] Cruz, A. T., Geltemeyer, A. M., Starke, J. R., Flores, J. A., Graviss, E. A., and Smith, K. C. 2011. "Comparing the Tuberculin Skin Test and T-SPOT.TB Blood Test in Children.” Pediatrics 127 (1): e31-8.

[12] Hesseling, A. C., Schaaf, H. S., Gie, R. P., Starke, J. R., and Beyers, N. 2002. "A Critical Review of Diagnostic 
Approaches Used in the Diagnosis of Childhood Tuberculosis.” Int. J. Tuberc. Lung Dis. 6 (12): 1038-45.

[13] Marais, B. J., Gie, R. P., Hesseling, A. C., Schaaf, H. S., Lombard, C., Enarson, D. A., and Beyers, N. 2006. “A Refined Symptom-Based Approach to Diagnose Pulmonary Tuberculosis in Children.” Pediatrics 118 (5): e1350-9.

[14] Radford, A. J., Rothel, J. S., and Sberna, G. 2006. "Whole Blood IFN-Gamma Assay for Detecting TB in Children.” Thorax 61: 920-1.

[15] Mahomed, H., Hughes, E. J., Hawkridge, T., Minnies, D., Simon, E., Little, F., Hanekom, W. A., Geiter, L., and Hussey, G. D. 2006. “Comparison of Mantoux Skin Test with Three Generations of a Whole Blood IFN-Gamma Assay for Tuberculosis Infection” Int. J. Tuberc. Lung Dis. 10 (3):310-6.
[16] Roy, R. B., Sotgiu, G., Altet-Gómez, N., Tsolia, M., Ruga, E., Velizarova, S., and Kampmann, B. 2012. "Identifying Predictors of Interferon- $\gamma$ Release Assay Results in Pediatric Latent Tuberculosis: A Protective Role of Bacillus Calmette-Guérin?” Am. J. Respir. Crit. Care Med. 186 (4):378-84.

[17] Mandalakas, A. M., Detjen, A. K., Hesseling, A. C., Benedetti, A., and Menzies, D. 2011. "Interferon- $\gamma$ Release Assays and Childhood Tuberculosis: Systematic Review and Meta-Analysis.” Int. J. Tuberc. Lung Dis. 15 (8): 1018-32.

[18] Machingaidze, S., Wiysonge, C. S., Gonzalez-Angulo, Y., Hatherill, M., Moyo, S., Hanekom, W., and Mahomed, H. 2011. "The Utility of an Interferon $\gamma$ Release Assay for Diagnosis of Latent Tuberculosis Infection and Disease in Children: A Systematic Review and Meta-Analysis." Pediatr. Infect. Dis. J. 30 (8): 694-700. 